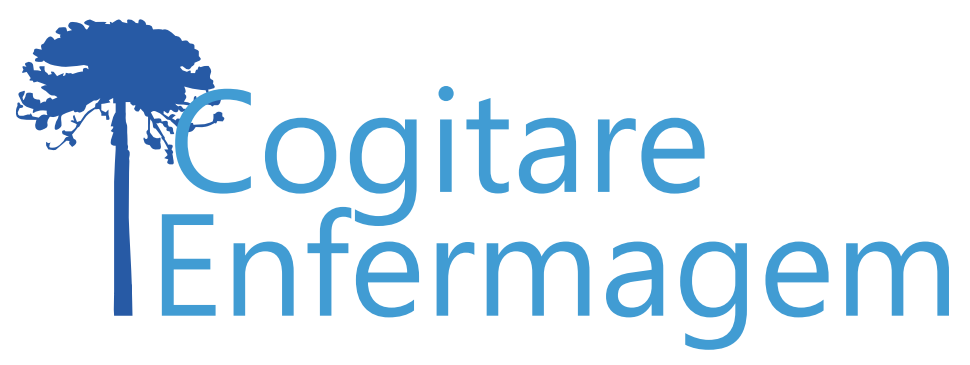

REVISÃO

\title{
IMAGEM GUIADA MELHORA A QUALIDADE DE VIDA RELACIONADA À SAÚDE DE PACIENTES COM CÂNCER? REVISÃO INTEGRATIVA
}

\author{
Luana Aparecida Alves da Silva ${ }^{1}$ (1) \\ Edenice de Oliveira Santana ${ }^{2}$ (i) \\ Larissa Marcondes ${ }^{2}$ (c) \\ Mariana Nunes da Silva ${ }^{3}$ (1) \\ Andressa Blitzkow Scherer ${ }^{3}$ (i) \\ Namie Okino Sawada ${ }^{4}$ (อ) \\ Luciana Puchalski Kalinke ${ }^{3}$ (c)
}

\section{RESUMO}

Objetivo: analisar pesquisas que utilizam Imagem Guiada como estratégia para melhoria da qualidade de vida relacionada à saúde de pacientes com câncer.

Método: revisão integrativa com avaliação do nível de evidência, realizada em fevereiro/2020, com publicações de janeiro/2009 a fevereiro/2020, da Biblioteca Virtual em Saúde, National Library of Medicine, Scopus e Web of Science, com os descritores: Imagens, Psicoterapia; Neoplasias e Qualidade de vida, em inglês, espanhol e português.

Resultados: 16 artigos foram publicados entre 2013 e 2019, 14 em inglês, 11 ensaios clínicos randomizados, incluindo diferentes tipos de câncer. A Imagem Guiada foi associada a técnicas como exercícios respiratórios e/ou relaxamento muscular progressivo. Resultados positivos para qualidade de vida relacionada à saúde foram observados em $75 \%$ dos estudos.

Conclusão: a imagem guiada apresenta benefícios quando utilizada para melhoria da qualidade de vida relacionada à saúde de pacientes com câncer. O presente estudo contribui para promoção e divulgação dessa prática.

DESCRITORES: Qualidade de Vida; Imagens, Psicoterapia; Neoplasias; Oncologia Integrativa; Terapias Mente-Corpo.

\section{¿LAS IMÁGENES GUIADAS MEJORAN LA CALIDAD DE VIDA RELACIONADA CON LA SALUD DE PACIENTES COM CÁNCER? UNA REVISIÓN INTEGRADORA}

\section{RESUMEN:}

Objetivo: analizar investigaciones que emplean Imágenes Guiadas como estrategia para mejorar la calidad de vida relacionada con la salud de pacientes con cáncer. Método: revisión integradora con evaluación del nivel de evidencia, realizada en febrero de 2020, con publicaciones de enero de 2009 a febrero de 2020, de la Biblioteca Virtual en Salud, la National Library of Medicine, Scopus y Web of Science, y con los siguientes descriptores: Imágenes, Psicoterapia; Neoplasias y Calidad de vida, en inglés, español y portugués. Resultados: se publicaron 16 artículos entre 2013 y 2019; 14 en inglés, y 11 fueron ensayos clínicos aleatorizados, incluyendo diferentes tipos de cáncer. Las Imágenes Guiadas se asociaron a técnicas como ser ejercicios respiratorios y/o relajación muscular progresiva. Se observaron resultados positivos para la calidad de vida relacionada con la salud en el $75 \%$ de los estudios. Conclusión: las imágenes guiadas presentan beneficios cuando se las emplea para mejorar la calidad de vida relacionada con la salud de pacientes con cáncer. Este estudio contribuye a la promoción y divulgación de esa práctica.

DESCRIPTORES: Calidad de Vida; Imágenes, Psicoterapia; Neoplasias; Oncología Integradora; Terapias MenteCuerpo. 
O câncer configura um dos principais problemas de saúde pública devido a sua incidência: foram estimados mais de 18 milhões de novos casos no mundo em $2018^{(1)}$. Com o diagnóstico e tratamento, várias experiências estressantes acompanham os pacientes e sobreviventes, podendo causar efeitos físicos, psicológicos e comportamentais, desencadeando preocupação com alterações na qualidade de vida (QV) e bem-estar dessas pessoas ${ }^{(2)}$.

Em resposta às necessidades crescentes dos pacientes na participação ativa e administração de seu próprio cuidado, promovendo melhora da condição de saúde, QV e empoderamento, a oncologia integrativa é uma área emergente ${ }^{(3)}$. As terapias integrativas e complementares têm sido utilizadas em centros de tratamentos oncológicos internacionais, tornando-se populares entre os pacientes ${ }^{(4)}$.

Dentre as terapias, as práticas mente-corpo, baseadas na ligação entre a mente e a saúde física, são utilizadas como auxílio na diminuição de efeitos colaterais e melhora da qualidade de vida relacionada à saúde $(\mathrm{QVRS})^{(3)}$. Técnicas e procedimentos podem ser utilizadas isoladas ou em conjunto. Entre elas estão Imagem Guiada, meditação, exercícios de respiração, relaxamento muscular progressivo (RMP) e hipnose ${ }^{(5)}$.

A Imagem Guiada é uma intervenção de aplicação simples e econômica, requer pouco esforço para o paciente e pode ser aplicada por enfermeiros, médicos e psicólogos em todos os estágios do câncer(b). Consiste em trabalhar mentalmente a visualização de imagens agradáveis e indução de pensamentos positivos, para substituir a negatividade e fatores estressantes ${ }^{(7)}$, e está entre as práticas mais pesquisadas ${ }^{(7-8)}$. Sua utilização é geralmente bem aceita pelos pacientes e seus efeitos são facilmente notados; além da recuperação física, há recuperação emocional do paciente ${ }^{(9)}$. A melhora no domínio emocional contribui para diminuir o impacto negativo na QVRS de pacientes com câncer, geralmente desencadeados após o início do tratamento ${ }^{(10)}$.

Assim, este estudo objetivou analisar pesquisas que utilizaram a Imagem Guiada como estratégia para melhoria da qualidade de vida relacionada à saúde de pacientes com câncer.

\section{MÉTODO}

Revisão integrativa de literatura que seguiu as etapas de pesquisa: definição do problema, formulação da questão norteadora; estabelecimento dos critérios de inclusão e exclusão; seleção e avaliação dos artigos; discussão dos resultados e apresentação da síntese ${ }^{(11)}$.

Para auxiliar na identificação de tópicos-chave e formulação da questão norteadora, foi utilizado o acrônimo PICO (Paciente, Intervenção, Comparação, Outcomes ou desfecho) (12). Sendo P-paciente com câncer, I-imagem guiada, C-sem comparação e O-qualidade de vida. Assim, a questão norteadora foi definida como: Os pacientes com câncer, durante seu tratamento, têm benefício com a utilização da Imagem Guiada como estratégia para melhoria da sua qualidade de vida relacionada à saúde?

As buscas foram realizadas em fevereiro de 2020, na Biblioteca Virtual em Saúde (BVS), National Library of Medicine (PubMed), Scopus e Web of Science. Os Descritores em Ciências da Saúde/Medical Subject Headings (DeCS/ MeSH) Imagens (Psicoterapia), Neoplasias e Qualidade de Vida/Imagery, Psychotherapy; Neoplasms e Quality of Life 
nos idiomas inglês, espanhol e português, utilizando o operador booleano "AND" foram adotados para a estratégia de busca. Optou-se por acrescentar as palavras-chave Imagens guiadas/Guided Imagery e Câncer/Cancer como sinônimos para o primeiro e segundo descritor respectivamente, utilizando o operador booleano "OR", a fim de ampliar os resultados (Quadro 1).

Quadro 1 - Estratégia de busca. Curitiba, PR, Brasil, 2020

\begin{tabular}{|c|c|}
\hline Bases & Estratégia \\
\hline Biblioteca Virtual em Saúde & \multirow{4}{*}{$\begin{array}{c}\text { ("Imagery (Psychotherapy)" OR "Imágenes (Psicoterapia)" OR "Imagens } \\
\text { (Psicoterapia)" OR "Imagery, Psychotherapy" OR "Guided Imagery" } \\
\text { OR "Imágenes Guiadas" OR "Imagens Guiadas") AND (Neoplasms OR } \\
\text { Neoplasias OR Cancer OR Câncer) AND ("Quality of Life" OR "Calidad } \\
\text { de Vida" OR "Qualidade de Vida") }\end{array}$} \\
\hline National Library of Medicine & \\
\hline Scopus & \\
\hline Web of Science & \\
\hline
\end{tabular}

Fonte: Autores (2020)

Os critérios de inclusão foram: artigos originais, disponíveis na íntegra, publicados no período de janeiro de 2009 até fevereiro de 2020. Foram excluídos artigos que não corresponderam à questão norteadora ou ao objetivo, artigos de revisão e outras publicações.

A seleção foi realizada por dois revisores independentemente, as discordâncias foram resolvidas por consenso com um terceiro revisor. Os artigos foram ordenados sequencialmente em planilha Microsoft Excel 365®, excluindo-se os repetidos, mantendose o primeiro registro. Para primeira avaliação, foi realizada leitura dos títulos e resumos para verificar a adequação à questão norteådora e objetivo.

Os artigos selecionados foram lidos na íntegra e extraídas informações relevantes em instrumento para categorização e mapeamento das informações: base de dados, periódico, ano de publicação, autores, categoria, idioma, título, objetivo, abordagem metodológica, local de estudo, participantes, descrição da técnica utilizada, duração da técnica, instrumento utilizado para avaliar QVRS, resultados, limitações, considerações, grau de recomendação (GR) e nível de evidência (NE). Estes últimos foram classificados pelo Oxford Centre for Evidence-based Medicine ${ }^{(13)}$, que apresenta um modelo hierárquico de $A$ a D para GR e 1 a 5 para NE, onde A e 1 possuem o maior valor.

\section{RESULTADOS}

A busca inicial resultou em 159 estudos. Após as exclusões, a composição foi de 16 artigos. A Figura 1 apresenta o fluxograma de seleção baseado no Preferred Reporting Items for Systematic Reviews and Meta-Analyses (PRISMA). 


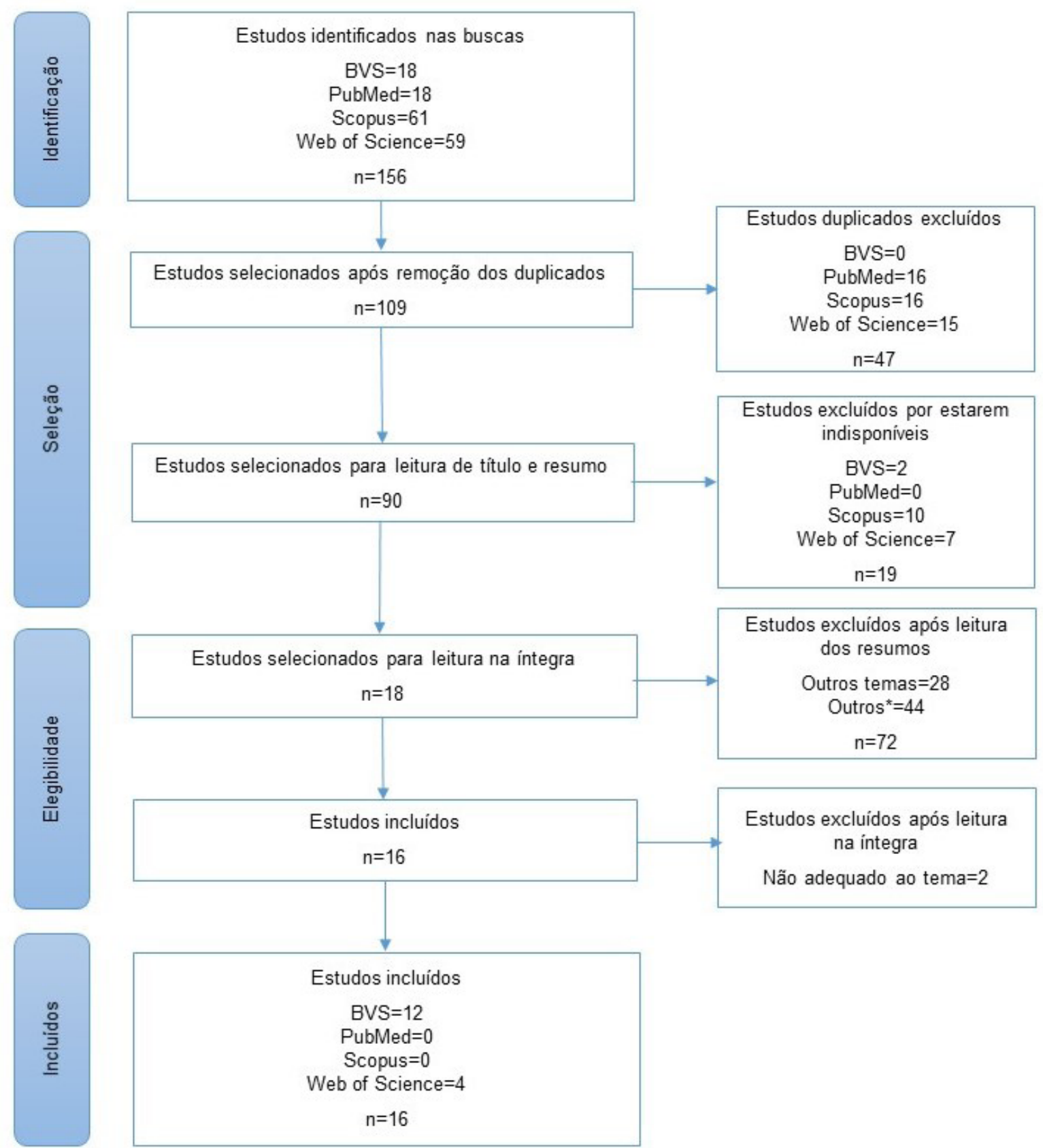

*Revisões $(n=28)$, editoriais $(n=4)$, capítulo de livro $(n=4)$, resumos $(n=3)$, artigos de opinião $(n=1)$, cartas $(n=1)$, reflexões $(n=1)$, publicações governamentais $(n=1)$, anais $(n=1)$.

Figura 1 - Fluxograma do processo de seleção. Curitiba, PR, Brasil, 2020

Fonte: Autores (2020)

Dos artigos selecionados, $14(87,5 \%)$ estão em inglês e dois em português. O ano de 2018 foi o mais expressivo com cinco (31,3\%) publicações. Profissionais de psicologia foram o autor principal em nove $(56,3 \%)$ artigos, seguido da enfermagem e medicina com cinco $(31,3 \%)$ e dois $(12,5 \%)$, respectivamente.

Em relação ao objetivo, 12 (75\%) estudos buscaram avaliar a eficácia de intervenções integrativas e complementares sobre a QVRS e/ou sintomas do paciente com câncer. Outros objetivos foram: compreender o significado da intervenção para os participantes, A1; avaliar viabilidade e aceitabilidade da intervenção, A2; apresentar resultados qualitativos de estudo prévio, A8; e promover transformações psicoespirituais e sociais, A12; nos quais a QV esteve implícita. O Quadro 2 apresenta os estudos selecionados. 
Quadro 2 - Estudos incluídos segundo identificação, tipo de pesquisa, número amostral, descrição da intervenção e resultados. Curitiba, PR, Brasil, 2020 (continua)

\begin{tabular}{|c|c|c|c|c|}
\hline ID* & $\begin{array}{l}\text { Tipo de } \\
\text { Pesquisa (n) }\end{array}$ & Descrição da intervenção & Resultados & $\begin{array}{l}\text { GR/ } \\
\text { NE** }\end{array}$ \\
\hline $\begin{array}{l}\text { A1 - Toneti BF, } \\
\text { et al. Rev Esc } \\
\text { Enferm USP. } \\
2019^{(14)}\end{array}$ & $\begin{array}{l}\text { Fenomenológico } \\
(\mathrm{n}=9)\end{array}$ & $\begin{array}{l}\text { Relaxamento com } \\
\text { imaginação guiada, RMP } \\
\text { conduzido em associação } \\
\text { à visualização de imagens, } \\
\text { CD-15 minutos. Intervenção } \\
\text { única. }\end{array}$ & $\begin{array}{l}\text { Melhora da QV durante o } \\
\text { tratamento. As participantes } \\
\text { relataram bem-estar e } \\
\text { relaxamento físico e mental } \\
\text { após as sessões. }\end{array}$ & $B / 2 C$ \\
\hline $\begin{array}{l}\text { A2 - } \\
\text { Trachtenberg } \\
\text { L, et al. } \\
\text { Psychooncology. } \\
2019^{(15)}\end{array}$ & $\begin{array}{l}\text { Estudo de } \\
\text { viabilidade } \\
(n=60)\end{array}$ & $\begin{array}{l}\text { Exercícios de imagem } \\
\text { guiada uma vez por semana, } \\
\text { material psicoeducacional e } \\
\text { processos psicoterapêuticos } \\
\text { grupais. Duração de oito } \\
\text { semanas. }\end{array}$ & $\begin{array}{l}\text { Identificou melhora na QV, } \\
\text { sem significância }\end{array}$ & $\mathrm{B} / 2 \mathrm{~B}$ \\
\hline $\begin{array}{l}\text { A3 - Barre PV, } \\
\text { et al. Indian J } \\
\text { Psychol Med. } \\
2018^{(17)}\end{array}$ & $\begin{array}{l}\text { Pré } \\
\text { experimental, } \\
\text { grupo único } \\
\text { pré e pós teste } \\
(n=30)\end{array}$ & $\begin{array}{l}\text { Pacote psicológico de } \\
\text { psicoeducação, relaxamento } \\
\text { com imagem guiada } \\
\text { e terapia cognitivo- } \\
\text { comportamental. Duração de } \\
\text { seis semanas. }\end{array}$ & $\begin{array}{l}\text { Estado de saúde global/ } \\
\text { OV melhor pós-intervenção. } \\
\text { Significância entre escores } \\
\text { pré e pós-intervenção em: } \\
\text { função física, emocional e } \\
\text { desempenho de papeis. } \\
\text { Diferenças significativas } \\
\text { nos sintomas: fadiga, dor, } \\
\text { dispneia, insônia, perda } \\
\text { de apetite, constipação e } \\
\text { diarreia. }\end{array}$ & $\mathrm{B} / 2 \mathrm{~B}$ \\
\hline $\begin{array}{l}\text { A4 - Esplen } \\
\text { MJ, et al. J Clin } \\
\text { Oncol. } 2018^{(18)}\end{array}$ & $\begin{array}{l}\mathrm{ECR}^{\star \star \star \star *}(\mathrm{n}=194- \\
\mathrm{Gl}=131, \mathrm{GC}=63)\end{array}$ & $\begin{array}{l}\text { Sessões semanais de } 90 \\
\text { minutos com: imagens, } \\
\text { psicoterapia e informação. } \\
\text { Duração de oito semanas. }\end{array}$ & $\begin{array}{l}\text { Mudança na QV não foi } \\
\text { significativa. A subescala } \\
\text { Breast mostrou melhora } \\
\text { no grupo de intervenção } \\
\text { com } 12 \text { meses de } \\
\text { acompanhamento. A } \\
\text { interação grupo por tempo } \\
\text { foi significativa. }\end{array}$ & $A / 1 B$ \\
\hline $\begin{array}{l}\text { A5 - Hoogland } \\
\text { Al, et al. } \\
\text { Psychooncology. } \\
2018^{(19)}\end{array}$ & $\begin{array}{l}\mathrm{ECR}(\mathrm{n}=240- \\
\mathrm{Gl}=121 \\
\mathrm{GC}=119)\end{array}$ & $\begin{array}{l}\text { Gerenciamento do estresse } \\
\text { durante quimioterapia } \\
\text { com: respiração abdominal, } \\
\text { RMP/imagem guiada e } \\
\text { autoafirmações. Duração de } \\
13 \text { semanas. }\end{array}$ & $\begin{array}{l}\text { O bem-estar emocional } \\
\text { melhorou significativamente. } \\
\text { O bem-estar funcional } \\
\text { e físico diminuíram } \\
\text { significativamente. A } \\
\text { gravidade total dos } \\
\text { sintomas aumentou } \\
\text { significativamente. }\end{array}$ & $A / 1 B$ \\
\hline $\begin{array}{l}\text { A6 - Nicolussi } \\
\text { AC, et al. Rev } \\
\text { Enferm Atenção } \\
\text { Saúde. } 2018^{(20)}\end{array}$ & $\begin{array}{l}\text { Quase- } \\
\text { experimental, } \\
(\mathrm{n}=152-\mathrm{G}=73 \\
\mathrm{GC}=79)\end{array}$ & $\begin{array}{l}\text { Gravação em CD com } \\
\text { duração de } 15 \text { minutos. } \\
\text { Aplicado durante o } \\
\text { tratamento quimioterápico. }\end{array}$ & $\begin{array}{l}\text { Em T2 diferença significativa } \\
\text { para função física e no } \\
\text { T3 para as funções física, } \\
\text { emocional, desempenho } \\
\text { de papel, fadiga, náuseas/ } \\
\text { vômitos. Para o grupo } \\
\text { intervenção, houve aumento } \\
\text { na função emocional e } \\
\text { diminuição dos sintomas de } \\
\text { náuseas/vômitos. }\end{array}$ & $B / 2 B$ \\
\hline
\end{tabular}




\begin{tabular}{|c|c|c|c|c|}
\hline $\begin{array}{l}\text { A7 - Stoerkel E, } \\
\text { et al. J Altern } \\
\text { Complement } \\
\text { Med. } 2018^{(21)}\end{array}$ & $\begin{array}{l}\mathrm{ECR}(n=100- \\
\mathrm{Gl}=49, \mathrm{GC}=51)\end{array}$ & $\begin{array}{l}\text { Respiração, relaxamento, } \\
\text { meditação, imagens guiadas, } \\
\text { auto hipnose e ferramentas } \\
\text { (pulseiras de acupressão e } \\
\text { diário). Duração de duas } \\
\text { semanas. } \\
\end{array}$ & $\begin{array}{l}\text { Não houve diferença entre } \\
\text { os dois grupos do estudo. }\end{array}$ & $A / 1 B$ \\
\hline $\begin{array}{l}\text { A8 - Elias ACA, } \\
\text { et al. Estud. } \\
\text { psicol. } 2017^{(22)}\end{array}$ & $\begin{array}{l}\text { Exploratório } \\
\text { comparativo, } \\
\text { descritivo }(n=28)\end{array}$ & $\begin{array}{l}\text { Técnicas de relaxamento, } \\
\text { imagens guiadas e elementos } \\
\text { de espiritualidade. Duração } \\
\text { média de cinco semanas. }\end{array}$ & $\begin{array}{l}\text { Melhora significativa na QV } \\
\text { percebida. Não apresentou } \\
\text { melhora significativa nos } \\
\text { domínios do WHOQOL ou } \\
\text { na satisfação com a saúde, } \\
\text { segundo o WHOQOL. }\end{array}$ & $\mathrm{B} / 2 \mathrm{C}$ \\
\hline $\begin{array}{l}\text { A9 - Shahriari } \\
\text { M, et al. J Educ } \\
\text { Health Promot. } \\
2017^{(23)}\end{array}$ & $\begin{array}{l}E C^{\star \star \star}(n=50- \\
G I=25, G C=25)\end{array}$ & $\begin{array}{l}\text { Imagens de cenários } \\
\text { relaxantes ao menos duas } \\
\text { vezes/dia. Duração de seis } \\
\text { semanas. }\end{array}$ & $\begin{array}{l}\text { Aumento significativo no } \\
\text { grupo intervenção na QV } \\
\text { geral e domínios funcionais. }\end{array}$ & $A / 1 B$ \\
\hline $\begin{array}{l}\text { A10 - } \\
\text { Charalambous } \\
\text { A, et al. PLoS } \\
\text { One. } 2016^{(4)}\end{array}$ & $\begin{array}{l}\mathrm{ECR}(n=236 \\
\mathrm{GI}=120 \\
\mathrm{GC}=116)\end{array}$ & $\begin{array}{l}\text { Roteiro com imagens } \\
\text { auditivas, táteis e olfativas } \\
\text { acompanhado por música: } \\
\text { exercício respiratório, } \\
\text { seguido de RMP sessão de } \\
\text { imagens guiadas. Duração de } \\
\text { quatro semanas }\end{array}$ & $\begin{array}{l}\text { Aumento dos escores } \\
\text { globais de QV e melhor } \\
\text { QVRS no grupo intervenção. } \\
\text { Diminuição significativa } \\
\text { após a intervenção para } \\
\text { funcionamento sexual e } \\
\text { funções relacionadas ao } \\
\text { tratamento e aumento } \\
\text { significativo em todas as } \\
\text { pontuações funcionais. }\end{array}$ & $A / 1 B$ \\
\hline $\begin{array}{l}\text { A11 - Koplin G, } \\
\text { et al. Cancer } \\
\text { Med. } 2016^{(24)}\end{array}$ & $\begin{array}{l}E C R \\
(n=60-G 1=20, \\
G 2=22, G 3=18)\end{array}$ & $\begin{array}{l}\text { Áudio de } 12 \text { minutos três } \\
\text { vezes ao dia. Aplicação dois } \\
\text { dias antes da cirurgia e por } \\
\text { ao menos } 30 \text { dias após. }\end{array}$ & $\begin{array}{l}\text { O grupo intervenção teve } \\
\text { escores mais altos de perda } \\
\text { de apetite no terceiro dia } \\
\text { e de dispneia no } 30^{\circ} \text { dia } \\
\text { pós-operatório. No } 30^{\circ} \\
\text { dia pós-operatório, as } \\
\text { pontuações para a OV foram } \\
\text { nitidamente mais baixas nos } \\
\text { pacientes com ostomia. }\end{array}$ & $B / 2 B$ \\
\hline $\begin{array}{l}\text { A12 - Elias } \\
\text { AC, et al. } \\
\text { Complement } \\
\text { Ther Clin Pract. } \\
2015^{(16)}\end{array}$ & $\operatorname{ECR}(n=28)$ & $\begin{array}{l}\text { Técnicas de relaxamento, } \\
\text { imagens guiadas e elementos } \\
\text { de espiritualidade. Duração } \\
\text { de três semanas. }\end{array}$ & $\begin{array}{l}\text { Melhora significativa na } \\
\text { percepção de OV após } \\
\text { intervenção. Não houve } \\
\text { melhora significativa nos } \\
\text { domínios de QV ou na } \\
\text { satisfação com a saúde. } \\
\end{array}$ & $B / 2 B$ \\
\hline $\begin{array}{l}\text { A13 - Freeman } \\
\text { LW, et al. } \\
\text { Psychooncology. } \\
2015^{(25)}\end{array}$ & ECR $(n=118)$ & $\begin{array}{l}\text { CD com imagens guiadas, } \\
\text { de } 20 \text { a } 30 \text { minutos. Foi } \\
\text { encorajado práticas diárias. } \\
\text { Duração de ao menos três } \\
\text { meses. }\end{array}$ & $\begin{array}{l}\text { O grupo intervenção } \\
\text { apresentou melhora na } \\
\text { função cognitiva e fadiga, } \\
\text { assim como melhora } \\
\text { significativa na qualidade do } \\
\text { sono. }\end{array}$ & $B / 2 B$ \\
\hline $\begin{array}{l}\text { A14 - Garssen } \\
\text { B, et al. } \\
\text { Psychooncology. } \\
2013^{(26)}\end{array}$ & $\begin{array}{l}\mathrm{ECR}(n=70- \\
\mathrm{GI}=34, \mathrm{GC}=36)\end{array}$ & $\begin{array}{l}\text { Técnicas de imagem guiada } \\
\text { e aconselhamento, por meio } \\
\text { de áudio para ser usado em } \\
\text { casa. Duração de um mês. }\end{array}$ & $\begin{array}{l}\text { A QV aumentou nos dias } \\
\text { dois e } 30 \text { após a cirurgia } \\
\text { no grupo intervenção. Não } \\
\text { houve diferença significativa } \\
\text { entre os grupos. }\end{array}$ & $B / 1 B$ \\
\hline
\end{tabular}




\begin{tabular}{|c|c|c|c|c|}
\hline $\begin{array}{l}\text { A15 - Gaston- } \\
\text { Johansson } \\
\text { F, et al. } \\
\text { Psychooncology. } \\
2013^{(27)}\end{array}$ & ECR $(n=110)$ & $\begin{array}{l}\text { Comprehensive coping } \\
\text { strategy program com: } \\
\text { educação preparatória, } \\
\text { reestruturação cognitiva, } \\
\text { aprimoramento } \\
\text { de habilidades de } \\
\text { enfrentamento e } \\
\text { relaxamento com imagens } \\
\text { guiadas. Duração de ao } \\
\text { menos três meses. }\end{array}$ & $\begin{array}{l}\text { O grupo intervenção } \\
\text { apresentou melhora } \\
\text { significativa na QV geral, } \\
\text { saúde e funcionamento e } \\
\text { socioeconômico e bem-estar } \\
\text { psicológico/espiritual. }\end{array}$ & $A / 1 B$ \\
\hline $\begin{array}{l}\text { A16 - Jacobsen } \\
\text { PB, et al. } \\
\text { Psychooncology. } \\
2013^{(28)}\end{array}$ & ECR $(n=460)$ & $\begin{array}{l}\text { Vídeo, livreto e áudio com } \\
\text { informações, demonstrações } \\
\text { e instruções sobre } \\
\text { respiração controlada, RMP } \\
\text { com imagem guiada e } \\
\text { autoafirmações. Duração de } \\
12 \text { semanas. }\end{array}$ & $\begin{array}{l}\text { Nenhuma das condições } \\
\text { de intervenção teve um } \\
\text { impacto benéfico em } \\
\text { medidas compostas de QV } \\
\text { física e mental. }\end{array}$ & $B / 2 B$ \\
\hline
\end{tabular}

*Identificação do Estudo. ${ }^{\star \star}$ GR-Grau de recomendação/NE-Nível de evidência. ${ }^{\star \star \star}$ EC-Ensaio clínico. ${ }^{\star \star \star \star E C R-E n s a i o ~ C l i ́ n i c o ~}$ Randomizado.

Fonte: Autores (2020)

Os estudos quantitativos se destacam com 14 (87,5\%), sendo representados pelos ensaios clínicos randomizados com 11 (68,8\%). Referente ao GR e NE, sete (44\%) apresentaram nível A/1B.

Para avaliação da QVRS, os instrumentos da European Organization for Research and Treatment of Cancer (EORTC) foram utilizados em sete $(43,8 \%)$ artigos, com destaque para - Quality of Life Questionnaire Core 30 (QLQ-C30) utilizado integralmente nos artigos A3, A6, A9 e A11; associado a módulos específicos, A10; ou em parte por A7 e A14.

Os instrumentos do Functional Assessment of Chronic Illness Therapy (FACIT) Measurement System foram utilizados em quatro (25\%), tanto o Functional Assessment of Cancer Therapy-General (FACT-G) quanto instrumentos específicos para a doença ou sintoma, destacados pelos artigos A2, A4, A5 e A13.

O Short-Form Health Survey (SF-36) foi utilizado pelos artigos A13 e A16; o World Health Organization Quality of Life-bref (WHOQOL-bref) foi utilizado em A8 e A12. Outros instrumentos utilizados foram Gastrointestinal Quality of Life index (GIOLI), A11, e Quality of Life Index-Cancer Version (QOLI-CV), A15. O estudo qualitativo A1 utilizou entrevista fenomenológica. Dos 16 estudos, três utilizaram mais de um instrumento para avaliação da OVRS: A5, A11 e A13.

Foram incluídos participantes com diagnóstico de câncer antes do início do tratamento, durante e após o término. A Imagem Guiada foi aplicada por períodos diversos, desde aplicação única até 13 semanas; em sete $(43,8 \%)$ estudos, os participantes foram estimulados a realizar a intervenção diariamente.

Em todos os estudos, a Imagem Guiada foi utilizada associada a outras técnicas, mais comumente exercícios respiratórios e/ou RMP. Psicoterapia, educação em saúde ou psicoeducação, música, meditação, pulseira de acupressão, terapia cognitivocomportamental, elementos de espiritualidade, autoafirmações e aconselhamento também foram associados.

Os resultados de eficácia das intervenções na OVRS foram variados; 12 (75\%) identificaram melhora na QVRS percebida, Q̉V geral e/ou em domínios, funções ou 
sintomas. Destaca-se os estudos A2 e A14 que relataram melhora não significativa, e o A4 que apresenta melhora na avaliação com um instrumento específico, não havendo diferença pela avaliação com o instrumento genérico. Os estudos A5, A7 e A16 não apresentaram diferença ou melhora dos aspectos da QVRS. O estudo A11 identificou maiores escores de perda de apetite e dispneia no grupo submetido à Imagem Guiada, os pacientes ostomizados que receberam a intervenção tiveram escores mais baixos de QVRS avaliada pelo GIQLI. Efeitos adversos relacionados à Imagem Guiada não foram relatados.

\section{DISCUSSÃO}

Nesta revisão, foram encontradas situações e aplicações diversas da Imagem Guiada em pacientes com câncer. Além do impacto na QVRS, imagem corporal (A2, A4) e função sexual (A4), estresse (A3, A14, A15) e ansiedade (A7), promoção de transformações sociopsicoespirituais, autoestima, desesperança (A8, A12), sintomas (A10) e avaliação dos efeitos psicológicos da intervenção (A14), foram trabalhados com Imagem Guiada.

A quantidade e frequência das sessões de intervenção realizadas não estão descritas em todos os estudos, variando em relação à aplicação. Há descrição de estímulo e disponibilização de materiais para a realização da intervenção em casa, buscando aumentar a exposição do participante para alcance dos objetivos, contudo esse método não permite conhecer de fato se a terapia foi realizada, o número de vezes ou a qualidade da sessão. O número de intervenções pode ter influência no desfecho, sendo que a prática regular e duradoura tende a apresentar melhores resultados ${ }^{(29)}$, entretanto, em relação à QVRS, não foram encontradas evidências que suportem essa hipótese.

O principal motivo para o emprego da técnica foi a melhoria da QVRS e alívio de sintomas. Há evidências de que a terapia de relaxamento, que inclui o RMP e Imagem Guiada ou técnicas de visualização, adicionados ao tratamento padrão, reduz sintomas depressivos e distúrbios do humor e pode ter outros benefícios à saúde, incluindo resultados psicológicos que podem ser afetados pelo estresse e ansiedade ${ }^{(8,30)}$.

Houve ainda pontos positivos relatados pelos pacientes. Os artigos apontam sensação de leveza, bem-estar e relaxamento físico e mental (A1); diminuição significativa do estresse (A3), de náusea e vômito (A10); redução de fadiga e depressão (A10, $A 14, A 15)$; melhora significativa na autoestima e bem-estar (A12). A intervenção estimulou reações emocionais e ajudou mulheres a entenderem seus sentimentos (A4); promoveu empoderamento (A8). $O$ estudo $A 2$ relata que as participantes se consideraram muito satisfeitas $(73 \%, n=32)$ ou satisfeitas $(20 \%, n=$ nove) com a intervenção, relatando sensação de alívio $(82 \%, n=36)$ e apontando redução significativa na angústia relacionada à imagem corporal.

Nos estudos que não apresentaram resultados significativos ou positivos em relação à QVRS, houve resultados relevantes em outros aspectos. No A7, os autores concluem que a intervenção pode fornecer um método saudável de enfrentamento e aumento da resiliência durante o tratamento. Neste estudo, 94\% dos participantes se declararam satisfeitos ou muito satisfeitos, houve redução não significativa na ansiedade e significativa na dor. Além disso, os autores sugerem que o aumento abrupto da taxa de sedimentação de eritrócitos no pós-operatório, no grupo intervenção, implica em melhora na cicatrização de feridas. No estudo A14, os participantes relatam alta apreciação em relação à Imagem Guiada, e os resultados demonstram diminuição significativa da depressão e fadiga. Já o estudo A5 relata melhoria no bem-estar emocional.

A associação da Imagem Guiada com outras técnicas de relaxamento foi observada nos estudos, predominantemente exercícios respiratórios e/ou RMP. Eles têm a intenção de induzir estado de relaxamento, para que o indivíduo possa conceber imagens mentais tranquilizantes e confortantes ${ }^{(6)}$. A aplicação de mais de uma técnica concorrente apresenta 
melhores efeitos comparado com utilização isolada(23). Uma revisão sistemática, que objetivou avaliar se existe base de evidências que suporte o uso de terapias integrativas para aplicação clínica no contexto do câncer de mama, aponta que a utilização da Imagem Guiada para relaxamento está frequentemente associada ao $\mathrm{RMP}^{(30)}$, corroborando com os resultados encontrados.

Em relação à avaliação da $\mathrm{QV}$, observou-se a utilização de instrumentos validados e reconhecidos internacionalmente, genéricos e específicos nos diferentes estudos. Como não há um instrumento de avaliação de OVRS adequado para todas as situações, a comparação entre eles torna-se difícil. Para a escolha, deve-se observar se o que está sendo medido é representado pelo instrumento selecionado no momento da medição. Neste sentido, seja na prática clínica ou em pesquisa, é importante que os profissionais de saúde estejam atualizados sobre a qualidade dos instrumentos de medida de QVRS para fazer uma seleção mais adequada ${ }^{(31)}$.

Os instrumentos mais utilizados nos estudos foram QLQ-C30 e FACT-G, escalas multidimensionais construídas especificamente para avaliar QVRS em pacientes com câncer; adicionalmente, podem ser utilizadas subescalas específicas para tipos de câncer, tratamentos ou sintomas. O QLQ-C30 restringe seus itens a aspectos relativamente objetivos do funcionamento, enquanto o FACT-G incentiva o paciente a refletir sobre seus pensamentos e sentimentos ${ }^{(32)}$. A literatura destaca que instrumentos específicos são mais sensíveis para captar as mudanças provocadas pela doença e tratamento ${ }^{(33)}$.

OWHOQOL-bref é um instrumento multidimensional desenvolvido pela Organização Mundial da Saúde, abrangente porém genérico, e valoriza a percepção individual, podendo avaliar a QV de diferentes grupos e situações ${ }^{(34)}$. O SF-36 é um instrumento genérico de avaliação de QVRS, projetado para medir estados gerais de saúde e permitir comparações entre pacientes com diferentes doenças e a população em geral ${ }^{(35)}$. O GIQLI é específico para sintomas gastrintestinais, não necessariamente relacionados a câncer, sendo usado em gastroenterologia e cirurgia digestiva(36).

Os artigos encontrados nesta revisão apresentaram resultados positivos para alívio de sintomas e melhora na QVRS percebida, geral ou nos domínios afetados pelo câncer, relacionados à Imagem Guiada nas diversas fases do tratamento e na sobrevida.

O baixo número de publicações na temática pode ser uma limitação do estudo, assim como a heterogeneidade dos estudos em relação à metodologia e intervenção, como variações de roteiro, duração, associação com outras técnicas e número de aplicações da intervenção, que dificultam a generalização e a comparação dos resultados.

\section{CONSIDERAÇÕES FINAIS}

As evidências encontradas nesta revisão mostraram que a Imagem Guiada é uma estratégia que melhora a OVRS de pacientes com câncer, e pode ser recomendada por profissionais de saúde, tendo baixo custo e sem efeitos colaterais associados.

Muitos são os fatores que interferem nos resultados dos estudos analisados, a considerar: os diferentes tipos de câncer, abordagens terapêuticas, número de aplicações, estadiamento e prognóstico dos pacientes. Todavia, os estudos analisados demonstram que pacientes com câncer têm alteração na QVRS, assim, a utilização da Imagem Guiada, associada a outras técnicas, demonstrou melhora nos domínios, funções ou sintomas, apresentando impacto positivo na QVRS percebida e geral.

O presente estudo contribui para a promoção da utilização da Imagem Guiada para pacientes com câncer e para divulgação de evidểncias sobre os benefícios da prática. 
Agradecemos a Fundação Araucária - Apoio e Desenvolvimento Científico e Tecnológico do Paraná, pelos recursos disponibilizados no edital CP15-2017.

\section{REFERÊNCIAS}

1. Ferlay J, Colombet M, Soerjomataram I, Mathers C, Parkin DM, Piñeros M, et al. Estimating the global cancer incidence and mortality in 2018: GLOBOCAN sources and methods. Int. J. Cancer. [Internet]. 2019 [acesso em 26 maio 2020]; 144(8). Disponível em: https://onlinelibrary.wiley.com/doi/epdf/10.1002/ ijc.31937.

2. Miller KD, Nogueira L, Mariotto AB, Rowland JH, Yabroff KR, Alfano CM, et al. Cancer treatment and survivorship statistics, 2019. CA Cancer J Clin. [Internet]. 2019 [acesso em 26 maio 2020]; 69(5). Disponível em: https://acsjournals.onlinelibrary.wiley.com/doi/epdf/10.3322/caac.21565.

3. Grant SJ, Hunter J, Seely D, Balneaves LG, Rossi E, Bao T. Integrative Oncology: International Perspectives. Integr. Cancer Ther [Internet]. 2019 [acesso em 26 maio 2020]; 18(1). Disponível em: https:// journals.sagepub.com/doi/pdf/10.1177/1534735418823266.

4. Charalambous A, Giannakopoulou M, Bozas E, Marcou Y, Kitsios P, Paikousis L. Guided imagery and progressive muscle relaxation as a cluster of symptoms management intervention in patients receiving chemotherapy: a randomized control trial. PLoS ONE. [Internet]. 2016 [acesso em 27 maio 2020]; 11(6). Disponível em: https://www.ncbi.nlm.nih.gov/pmc/articles/PMC4920431/pdf/pone.0156911.pdf.

5. Satija A, Bhatnagar S. Complementary therapies for symptom management in cancer patients. Indian J Palliat Care. [Internet]. 2017 [acesso em 08 fev 2020]; 23(4). Disponível em: https://www.ncbi.nlm.nih.gov/ pmc/articles/PMC5661353/.

6. Coelho A, Parola V, Fernandes O, Querido A, Apóstolo J. Construção de um programa de imaginação guiada para doentes internados em unidades de cuidados paliativos. Rev Enf Ref. [Internet]. 2018 [acesso em 22 maio 2020]; IV(17). Disponível em: http://www.scielo.mec.pt/pdf/ref/vserlVn17/serlVn17a03.pdf.

7. Nicolussi AC, Sawada NO, Cardozo FMC, Paula JM de. Relaxamento com imagem guiada e presença de depressão em Pacientes com câncer durante quimioterapia. Cogit. Enferm. [Internet]. 2016 [acesso em 08 fev 2020]; 21(4). Disponível em: http://docs.bvsalud.org/biblioref/2017/04/833107/48208-191971-1pb.pdf.

8. Carlson LE, Zelinski E, Toivonen K, Flynn M, Qureshi M, Piedalue KA, et al. Mind-Body Therapies in Cancer: What Is the Latest Evidence?. Curr Oncol Rep. [Internet]. 2017 [acesso em 08 fev 2020]; 19(67). Disponível em: https://link.springer.com/content/pdf/10.1007/s11912-017-0626-1.pdf.

9. Paula JM de, Baviera AF, Toneti BF, Barbosa RM, Okino L, Sawada NO. Integrative therapies in cancer patients: a look at the experience in a university extension group in Brazil. Hos Pal Med Int Jnl. [Internet]. 2018 [acesso em 26 maio 2020]; 2(6). Disponível em: https://medcraveonline.com/HPMIJ/ HPMIJ-02-00134.pdf.

10. Binotto $M$, Schwartsmann G. Qualidade de vida relacionada à saúde de pacientes com câncer de mama: revisão integrativa da literatura. Rev. bras. cancerol [Internet]. 2020 [acesso em 24 maio 2020]; 66(1). Disponível em: https://doi.org/10.32635/2176-9745.RBC.2020v66n1.405.

11. Mendes KDS, Silveira RC de CP, Galvão CM. Revisão integrativa: método de pesquisa para a incorporação de evidências na saúde e na enfermagem. Texto contexto-enferm. [Internet]. 2008 [acesso em 02 fev 2020]; 17(4). Disponível em: http://dx.doi.org/10.1590/S0104-07072008000400018. 
12. Nobre MRC, Bernardo WM, Jatene FB. A prática clínica baseada em evidências: parte III avaliação crítica das informações de pesquisas clínicas. Rev Assoc Med Bras. [Internet]. 2004 [acesso em 02 mar 2021]; 50(2). Disponível em: https://doi.org/10.1590/S0104-42302004000200042.

13. Oxford Centre for Evidence-based Medicine: levels of evidence [Internet]; 2009. Disponível em: http://www.cebm.net/oxford-centre-evidence-based-medicine-levels-evidence-march-2009.

14. Toneti BF, Avelar JM de P, Sousa FH, Toneti AN, Sonobe HM, Sawada NO. O significado de uma terapia integrativa de relaxamento guiado para mulheres com câncer de mama. Rev Esc Enferm USP. [Internet]. 2019 [acesso em 21 maio 2020]; 53:e03497. Disponível em: http://dx.doi.org/10.1590/S1980$220 \times 2018024103497$.

15. Trachtenberg L, Wong J, Rennie H, McLeod D, Leung Y, Warner E, et al. Feasibility and acceptability of i-Restoring Body Image after Cancer (i-ReBIC): a pilot trial for female cancer survivors. Psychooncology. [Internet]. 2019 [acesso em 23 maio 2020]; 29(4). Disponível em: https://pubmed.ncbi.nlm.nih. gov/31724261/.

16. Elias ACA, Ricci MD, Rodriguez LHD, Pinto SD, Giglio JS, Baracat EC. The biopsychosocial spiritual model applied to the treatment of women with breast cancer, through RIME intervention (relaxation, mental images, spirituality). Complement Ther Clin Pract. [Internet]. 2015 [acesso em 23 maio 2020]; 21(1). Disponível em: https://pubmed.ncbi.nlm.nih.gov/25682524/.

17. Barre PV, Padmaja G, Rana S, Tiamongla. Stress and quality of life in cancer patients: medical and Psychological Intervention. Indian J Psychol Med. [Internet]. 2018 [acesso em 23 maio 2020]; 40(3). Disponível em: https://www.ncbi.nlm.nih.gov/pmc/articles/PMC5968644/.

18. Esplen MJ. Wong J, Warner E, Toner B. Restoring Body Image After Cancer (ReBIC): Results of a Randomized Controlled Trial. J Clin Oncol. [Internet]. 2018 [acesso em 23 maio 2020]; 36(8). Disponível em: https://pubmed.ncbi.nlm.nih.gov/29356610/.

19. Hoogland Al, Lechner SC, Gonzalez BD, Small BJ, Tyson DM, Asvat Y, et al. Efficacy of a spanish-language self-administered stress management training intervention for latinas undergoing chemotherapy. Psychooncology. [Internet]. 2018 [acesso em 23 maio 2020]; 27(4). Disponível em: https:// pubmed.ncbi.nlm.nih.gov/29462503/.

20. Nicolussi AC, Cardozo FMC, Paula JM de, Sawada NO. Relaxamento com imagem guiada e qualidade de vida relacionada à saúde de pacientes durante quimioterapia. Rev Enferm Atenção Saúde. [Internet]. 2018 [acesso em 23 maio 2020]; 7(2). Disponível em: http://seer.uftm.edu.br/revistaeletronica/index.php/ enfer/article/view/2130.

21. Stoerkel E, Bellanti D, Paat C, Peacock K, Aden J, Setlik R, et al. Effectiveness of a self-care toolkit for surgical breast cancer patients in a military treatment facility. J Altern Complement Med. [Internet]. 2018 [acesso em 23 maio 2020]; 24(9-10). Disponível em: https://www.ncbi.nlm.nih.gov/pmc/articles/ PMC6157373/.

22. Elias ACA, Ricci MD, Rodriguez LHD, Pinto SD, Giglio JS, Baracat EC. Development of a Brief Psychotherapy modality entitled RIME in a hospital setting using alchemical images. Estud. psicol. [Internet]. 2017 [acesso em 23 maio 2020]; 34(4). Disponível em: https://www.scielo.br/pdf/estpsi/ v34n4/1982-0275-estpsi-34-04-00534.pdf.

23. Shahriari M, Dehghan M, Pahlavanzadeh S, Hazini A. Effects of progressive muscle relaxation, guided imagery and deep diaphragmatic breathing on quality of life in elderly with breast or prostate cancer. J Educ Health Promot. [Internet]. 2017 [acesso em 23 maio 2020]; 6(1). Disponível em: https://pubmed.ncbi. nlm.nih.gov/28546966/.

24. Koplin G, Müller V, Heise G, Pratschke J, Schwenk W, Haase O. Effects of psychological interventions and patients' affect on short-term quality of life in patients undergoing colorectal surgery. Cancer Med. [Internet]. 2016 [acesso em 23 maio 2020]; 5(7). Disponível em: https://www.ncbi.nlm.nih.gov/pmcl articles/PMC4863926/. 
25. Freeman LW, White R, Ratcliff CG, Sutton S, Stewart M, Palmer JL, et al. A randomized trial comparing live and telemedicine deliveries of an imagery-based behavioral intervention for breast cancer survivors: reducing symptoms and barriers to care. Psychooncology. [Internet]. 2015 [acesso em 23 maio 2020]; 24(8). Disponível em: https://pubmed.ncbi.nlm.nih.gov/25146413/.

26. Garssen B, Boomsma MF, Meezenbroek E de J, Porsild T, Berkhof J, Berbee M, et al. Stress management training for breast cancer surgery patients. Psychooncology. [Internet]. 2013 [acesso em 23 maio 2020]; 22(3). Disponível em: https://pubmed.ncbi.nlm.nih.gov/22383279/.

27. Gaston-Johansson F, Fall-Dickson JM, Nanda JP, Sarenmalm EK, Browall M, Goldstein N. Long-term effect of the self-management comprehensive coping strategy program on quality of life in patients with breast cancer treated with high-dose chemotherapy. Psychooncology. [Internet]. 2013 [acesso em 23 maio 2020]; 22(3). Disponível em: https://pubmed.ncbi.nlm.nih.gov/22290808/.

28. Jacobsen PB, Phillips KM, Jim HSL, Small BJ, Faul LA, Meade CD, et al. Effects of self-directed stress management training and home-based exercise on quality of life in cancer patients receiving chemotherapy: a randomized controlled trial. Psychooncology. [Internet]. 2013 [acesso em 23 maio 2020]; 22(6). Disponível em: https://pubmed.ncbi.nlm.nih.gov/22715124/.

29. Cooley SJ, Williams SE, Burns VE, Cumming J. Methodological variations in guided imagery interventions using movement imagery scripts in sport: a systematic review. Journal of Imagery Research in Sport and Physical Activity. [Internet]. 2013 [acesso em 24 maio 2020]; 8(1). Disponível em: https://doi. org/10.1515/jirspa-2012-0005.

30. Greenlee H, DuPont-Reyes MJ, Balneaves LG, Carlson LE, Cohen MR, Deng G, et al. Clinical practice guidelines on the evidence-based use of integrative therapies during and after breast cancer treatment. CA Cancer J Clin. [Internet]. 2017 [acesso em 08 fev 2020]; 67(3). Disponível em: https://acsjournals. onlinelibrary.wiley.com/doi/epdf/10.3322/caac.21397.

310. Roij JV, Fransen H, Poll-Franse LV de, Zijlstra M, Raijmakers N. Measuring health-related quality of life in patients with advanced cancer: a systematic review of self-administered measurement instruments. Qual Life Res. [Internet]. 2018 [acesso em 22 maio 2020]; 27(8). Disponível em: https://pubmed.ncbi.nlm. nih.gov/29427216/.

32. Iravani K, Jafari $P$, Akhlaghi A, Khademi B. Assessing whether EORTC QLQ-30 and FACT-G measure the same constructs of quality of life in patients with total laryngectomy. Health Qual Life Outcomes. [Internet]. 2018 [acesso em 22 maio 2020]; 16(1). Disponível em: https://pubmed.ncbi.nlm.nih. gov/30217200/.

33. Sawada N, Toneti BF, Avelar JMP, Reis LS, Therrier S, Mendonça CL, et al. Qualidade de vida relacionada à saúde nas doenças crônicas não transmissíveis. In: Rezende EG, Fava SMCL, organizadores. Cronicidade na perspectiva multiprofissional. Alfenas: Editora Universidade Federal de Alfenas; 2020. p. 65-101.

34. Almeida-Brasil CC, Silveira MR, Silva KR, Lima MG, Faria CDC de M, Cardoso CL, et al. Qualidade de vida e características associadas: aplicação do WHOQOL-BREF no contexto da Atenção Primária à Saúde. Cien Saude Colet [Internet]. 2017 [acesso em 20 maio 2020]; 22(5). Disponível em: http://doi. org/10.1590/1413-81232017225.20362015.

35. Witt $S$, Krauss E, Barbero MAN, Muller V, Bonniaud P, Vancheri C, et al. Psychometric properties and minimal important differences of SF-36 in Idiopathic Pulmonary Fibrosis. Respir. Res. [Internet]. 2019 [acesso em 22 maio 2020]; 20(47). Disponível em: https://doi.org/10.1186/s12931-019-1010-5.

36. Rodríguez CT, Moreno BN, Sanguineti MA, Abedrapo MM, Azolas MR, Bocic AG, et al. Adaptación transcultural del cuestionario de calidad de vida gastrointestinal GIQLI (Gastrointestinal Quality of Life Index) para su aplicación en pacientes operados del aparato gastrointestinal. Rev. cir. [Internet]. 2019 [acesso em 25 maio 2020]; 71(4). Disponível em: https://scielo.conicyt.cl/scielo.php?script=sci arttext\&pid=S2452-45492019000400287\&lng=es. 


\section{COMO REFERENCIAR ESTE ARTIGO:}

Silva LAA da, Santana E de O, Marcondes L, Silva MN da, Andressa Blitzkow Scherer, Namie Okino Sawada, et al. Imagem guiada melhora a qualidade de vida relacionada à saúde de pacientes com câncer? Revisão integrativa. Cogit. Enferm. [Internet]. 2021 [acesso em "colocar data de acesso, dia, mês abreviado e ano"]; 26. Disponível em: http://dx.doi.org/10.5380/ce.v26i0.75334.

Recebido em: 24/07/2020

Aprovado em: 24/03/2021

Editora associada: Luciana Alcântara Nogueira

Autor Correspondente:

Luana Aparecida Alves da Silva

Universidade Federal do Paraná - Curitiba, PR, Brasil

E-mail: luanamcr@yahoo.com.br

Contribuição dos autores:

Contribuições substanciais para a concepção ou desenho do estudo; ou a aquisição, análise ou interpretação de dados do estudo - LAAS, EOS, LM, MNS, LPK

Elaboração e revisão crítica do conteúdo intelectual do estudo - LAAS, EOS, LM, MNS, ABS, NOS, LPK

Aprovação da versão final do estudo a ser publicado - LAAS, EOS, LM, NOS, LPK

Responsável por todos os aspectos do estudo, assegurando as questões de precisão ou integridade de qualquer parte do estudo - LAAS, EOS, LM, MNS, ABS, NOS, LPK

ISSN 2176-9133

Copyright $\odot 2021$ Este é um artigo em acesso aberto distribuído nos termos da Licença Creative Commons Atribuição, que permite o uso irrestrito, a distribuição e reprodução em qualquer meio desde que o artigo original seja devidamente citado. 